\title{
Relativistic Generalizations of the Quantum Harmonic Oscillator
}

\author{
A. Poszwa
}

Department of Physics and Computer Methods, University of Warmia and Mazury in Olsztyn, Słoneczna 54, 10-710 Olsztyn, Poland

(Received March 27, 2014)

We investigate the dynamics of the spin-less relativistic particle subject to an external field of a harmonic oscillator potential. The Klein-Gordon equation with one- and three-dimensional vector and scalar parabolic potentials is solved using the expansion of the wavefunction in properly selected basis-sets. The resonance states are determined using the complex coordinate rotation method. The analytic expressions for the first- and secondorder relativistic energy corrections are derived perturbatively. The relativistic model of the harmonic oscillator in the momentum representation, originally proposed by Znojil, is also discussed.

DOI: $10.12693 /$ APhysPolA.126.1226

PACS: 03.65.- $-\mathrm{w}$

\section{Introduction}

Relativistic generalizations of the harmonic oscillator (HO) are neither unique nor trivial. In the classical mechanics the relativistic description of the HO leads to the frequency-amplitude dependence. A discussion of this subject and references to the relevant works may be found in a recent work by Belendéz et al. [1] where the analytical approximations to the oscillation period and the periodic solutions have been constructed. Studies on the quantum models of the HO, performed in the 1930s, demonstrated that quantum systems with electrostatic quadratic potential have no bound solutions $[2,3]$. Numerous studies on this subject may be represented by a set of references [4-16], far from being complete. The most commonly known model of the relativistic HO for a spin- $1 / 2$ particle is the Dirac oscillator, usually associated with the contribution by Moshinsky and Szczepaniak [7] but, in fact, formulated nearly 20 years earlier by Cook [4]. It has been discussed in many works, including [17-23]. Another approach, also related to the one by Cook [4], in which $\mathrm{HO}$ has been defined as a system described by an equation invariant with respect to the transformation $\mathrm{p} \leftrightarrow a \boldsymbol{r}$, where $\mathrm{p}$ and $\boldsymbol{r}$ stand, respectively, for the momentum and the coordinate operators and $a$ is a constant, has been applied to studies on both Klein-Gordon (KG) and Dirac oscillators in Refs. [11, 24, 25]. Some authors have studied the Dirac and KG equations with equal scalar and vector potentials using, in particular, the oscillator potentials [26]. A class of exact solutions of the mass-dependent $\mathrm{KG}$ equation has been obtained using an analytical iteration method [27].

The purpose of this paper is solving the $\mathrm{KG}$ equations with parabolic potentials in one- and three spatial dimensions using vector and scalar couplings [28]. The results are compared with a model of the $\mathrm{KG} \mathrm{HO}$ derived by Znojil from the spinless Salpeter equation [8]. As it is well known, the KG equation with quadratic electrostatic potential, referred to as the vector potential has no square-integrable solutions $[2,3]$. This consequence of the existence of the negative-energy continuum appears in some one-particle systems e.g. in the KG $\mathrm{HO}$ with the electrostatic potential, but not in the KG $\mathrm{HO}$ with the scalar potential, and is inevitable in the case of relativistic many-particle systems where it appears as a consequence of the so-called Brown-Ravenhall disease [29]. From several methods applicable to the evaluation of the energies and the widths of the autoionizing states (the resonances) [30] the complex coordinate rotation (CCR) has been selected. This method has been successfully used to solving autoionizing one-electron Dirac [31, 32] and two-electron Dirac-Coulomb [33] problems. To the author's knowledge, as yet, it was not applied in the case of the KG equation.

\section{Basic equations}

The stationary KG equation for a free particle reads [28]:

$$
c^{2} \mathrm{p}^{2} \Psi(\boldsymbol{r})=\left(\mathcal{E}_{\mathrm{K}}^{2}-m^{2} c^{4}\right) \Psi(\boldsymbol{r}),
$$

where all symbols have their usual meaning. The introduction of an external electrostatic potential $\mathrm{V}(\boldsymbol{r})$ and a scalar potential $\mathrm{S}(\boldsymbol{r})$ results in the substitution

$$
\mathcal{E}_{\mathrm{K}} \rightarrow \mathcal{E}_{\mathrm{K}}-\mathrm{V}(\boldsymbol{r}), \quad m c^{2} \rightarrow m c^{2}+\mathrm{S}(\boldsymbol{r}) .
$$

Consequently, the KG equation for a particle in the external field may be written as

$$
c^{2} \mathrm{p}^{2} \Psi=\left[\left(\mathcal{E}_{\mathrm{K}}-\mathrm{V}\right)^{2}-\left(m c^{2}+\mathrm{S}\right)^{2}\right] \Psi,
$$

By setting the origin of the energy scale at the rest mass of the particle, i.e. by the substitution

$$
\mathcal{E}_{\mathrm{K}}=\mathcal{E}+m c^{2}
$$

the last equation may be rewritten in a Schrödinger-like form

$$
\left(\frac{\mathrm{p}^{2}}{2 m}+\mathrm{U}\right) \Psi=\epsilon \Psi
$$

where the effective potential $U$ (energy-dependent in the vector case) is given by

$$
\mathrm{U}=\mathrm{S}+\mathrm{V}+\frac{1}{2 m c^{2}}\left(\mathrm{~S}^{2}-\mathrm{V}^{2}+2 \mathcal{E} \mathrm{V}\right)
$$




$$
\epsilon=\mathcal{E}\left(1+\frac{\mathcal{E}}{2 m c^{2}}\right) .
$$

In the one-dimensional case

$$
\mathrm{p}^{2}=-\hbar^{2} \frac{\mathrm{d}^{2}}{\mathrm{~d} z^{2}}
$$

If the external potentials are spherically symmetric then by the substitution

$$
\Psi(\boldsymbol{r})=\frac{F(r)}{r} Y_{l m}(\vartheta, \phi),
$$

Eq. (5) may be reduced to the one-dimensional radial equation with

$$
\mathrm{p}^{2}=-\hbar^{2}\left[\frac{\mathrm{d}^{2}}{\mathrm{~d} r^{2}}-\frac{l(l+1)}{r^{2}}\right] .
$$

\subsection{Vector KG harmonic oscillator}

Taking $\mathbf{S}=0$ and

$$
\mathrm{V}(z)=\frac{m \omega^{2} z^{2}}{2} \quad \text { or } \quad \mathrm{V}(r)=\frac{m \omega^{2} r^{2}}{2}
$$

we get, respectively, one-dimensional vector $\mathrm{KG} \mathrm{HO}$ or three-dimensional isotropic vector KG HO (VKG). Setting $\hbar=1$ and

$$
\begin{aligned}
& \zeta=z \sqrt{m \omega}, \quad \rho=r \sqrt{m \omega}, \quad \lambda=\frac{\omega}{m c^{2}}, \quad E=\frac{\mathcal{E}}{\omega}, \\
& w=E(2+\lambda E), \quad w_{1}=1+\lambda E,
\end{aligned}
$$

Eq. (5), in the one-dimensional case, becomes

$$
\left(\mathrm{W}_{\mathrm{V}}^{\zeta}-w\right) \Psi(\zeta)=0
$$

where

$$
\mathrm{W}_{\mathrm{V}}^{\zeta}=-\frac{\mathrm{d}^{2}}{\mathrm{~d} \zeta^{2}}+w_{1} \zeta^{2}-\frac{\lambda}{4} \zeta^{4}
$$

As one can see, for sufficiently large $z$ the negative quartic term always dominate. The effective potential $\mathrm{W}_{\mathrm{V}}^{\zeta}$ is parabolic and positive for small $z$ but diverges to minus infinity for large $z$ (see Fig. 1 and discussion in Ref. [11]). The motion of the particle is unlimited since it tunnels through the potential barrier. We can easily find that the wave function takes the asymptotic form

$$
\Psi(\zeta) \sim \mathrm{e}^{ \pm \frac{1}{6} \mathrm{i} \sqrt{\lambda} \zeta^{3}}
$$

Therefore all solutions of Eq. (11) are shape resonances.

The KG equation for the spherical HO reads

$$
\left(\mathrm{W}_{\mathrm{V}}^{\rho}-w\right) F(\rho)=0,
$$

where

$$
\mathrm{W}_{\mathrm{V}}^{\rho}=-\frac{\mathrm{d}^{2}}{\mathrm{~d} \rho^{2}}+\frac{l(l+1)}{\rho^{2}}+w_{1} \rho^{2}-\frac{\lambda}{4} \rho^{4} .
$$

This equation does not have bound solutions either since the centrifugal term does not influence the behavior of the effective potential in infinity.

\subsection{Scalar KG harmonic oscillator}

In this case $\mathrm{V}=0$. By taking

$$
\mathrm{S}(z)=\frac{m \omega^{2} z^{2}}{2} \quad \text { or } \mathrm{S}(r)=\frac{m \omega^{2} r^{2}}{2}
$$

we get scalar KG harmonic oscillators (SKG). The general form of the KG equations is the same as in Eqs. (11) and (14) except that $\mathrm{W}_{\mathrm{V}}^{\zeta}$ and $\mathrm{W}_{\mathrm{V}}^{\rho}$ have to be replaced, respectively, by

$$
\mathrm{W}_{\mathrm{S}}^{\zeta}=-\frac{\mathrm{d}^{2}}{\mathrm{~d} \zeta^{2}}+\zeta^{2}+\frac{\lambda}{4} \zeta^{4}
$$

and by

$$
\mathrm{W}_{\mathrm{S}}^{\rho}=-\frac{\mathrm{d}^{2}}{\mathrm{~d} \rho^{2}}+\frac{l(l+1)}{\rho^{2}}+\rho^{2}+\frac{\lambda}{4} \rho^{4} .
$$

In this case the effective potential behaves as an infinite quartic potential well - it approaches $+\infty$ for $\rho \rightarrow \infty$ and all states are bound.

\subsection{Equally mixed potentials}

In this case we define

$$
\mathrm{S}(z)=\mathrm{V}(z)=\frac{m \omega^{2} z^{2}}{4} \quad \text { or } \quad \mathrm{S}(r)=\mathrm{V}(r)=\frac{m \omega^{2} r^{2}}{4} \text {. }
$$

After using variables (10) and rescaling once again coordinates according to

$$
x=\sqrt{\Omega} \zeta \quad \text { or } y=\sqrt{\Omega} \rho, \text { where } \quad \Omega=\sqrt{1+\frac{\lambda E}{2}},
$$

corresponding KG equations may be transformed to the forms

$$
\begin{aligned}
& \left(-\frac{\mathrm{d}^{2}}{\mathrm{~d} x^{2}}+x^{2}\right) \Psi(x)=2 E \Omega \Psi(x) \\
& \left(-\frac{\mathrm{d}^{2}}{\mathrm{~d} y^{2}}+\frac{l(l+1)}{y^{2}}+y^{2}\right) F(y)=2 E \Omega F(y) .
\end{aligned}
$$

The exact energy eigenvalues satisfy the algebraic equation

$$
2 \lambda E^{3}+4 E^{2}-\varepsilon^{2}=0,
$$

where $\varepsilon=2 n+1$ or $\varepsilon=4 n+2 l+3$ for one-and threedimensional oscillators, respectively. Since energies corresponding to bound states should have real values, we can find that they are given by

$$
E=\sqrt[3]{-\delta+\sqrt{\Delta}}+\sqrt[3]{-\delta-\sqrt{\Delta}}-\frac{2}{3 \lambda}
$$

where

$$
\Delta=\frac{\varepsilon^{2}}{\lambda^{2}}\left(\frac{-4}{27 \lambda^{2}}+\frac{\varepsilon^{2}}{16}\right)
$$

and

$$
\delta^{2}=\frac{64}{27^{2} \lambda^{6}}-\frac{4 \varepsilon^{2}}{27 \lambda^{4}}+\frac{\varepsilon^{4}}{16 \lambda^{2}} .
$$

The wave functions of the KG harmonic oscillators with equally-mixed parabolic potentials, in variables $x$ or $y$, are the same as the nonrelativistic ones. The only difference is that they correspond to relativistic energies, given by (24).

\subsection{Znojil harmonic oscillator}

The HO Hamiltonian

$$
H=\sqrt{p^{2} c^{2}+m^{2} c^{4}}-m c^{2}+\frac{1}{2} m \omega^{2} r^{2},
$$

derived from the so-called spinless Salpeter equation, has been used by Znojil [8] in his definition of relativistic HO. This form of the HO is referred hereafter as Znojil harmonic oscillator ( $\mathrm{ZHO}$ ). The corresponding eigenvalue equation, in the momentum space has a Schrödinger-like 
form

$$
\begin{aligned}
& \frac{m \hbar^{2} \omega^{2}}{2}\left[-\frac{\mathrm{d}^{2}}{\mathrm{~d} p^{2}}+\frac{l(l+1)}{p^{2}}\right] \psi(p) \\
& \quad+\left(\sqrt{p^{2} c^{2}+m^{2} c^{4}}-m c^{2}\right) \psi(p)=\mathcal{E} \psi(p) .
\end{aligned}
$$

Both one-dimensional and radial forms of the last equation may be easily written and solved numerically using an arbitrary integration technique designed for solving the Schrödinger equation.

\section{Algebraic solutions}

In this section the solutions of the HO equations by algebraic methods are presented. The general form of the equation is

$$
(\mathrm{W}-w) \Phi=0 .
$$

In the algebraic approach the wave functions are expanded in a properly selected basis $\phi_{n}$ :

$$
\Phi=\sum_{n=0}^{\infty} c_{n} \phi_{n}
$$

and Eq. (29) is transformed to the algebraic eigenvalue problem. In order to apply numerical procedures, we define a reduced problem by terminating the series at some cutoff $n=N$. As a consequence, the pertinent algebraic KG equation is transformed to a system of $N+1$ linear equations, which can be written in the matrix form as

$$
[\boldsymbol{M}(\mathrm{W})-w \boldsymbol{I}] \boldsymbol{C}=0,
$$

where $\boldsymbol{I}$ is the unit matrix, $\boldsymbol{C}^{\mathrm{T}}=\left[\begin{array}{llll}c_{0}, & c_{1}, & \ldots, & c_{N}\end{array}\right]$, $\boldsymbol{M}$ is the algebraic representation of the operator $\mathrm{W}$ for which the eigenvalue problem has been defined. Depending on the case, the form of $\phi_{n}$ and the structure of $\mathrm{W}$ varies. Scalar and vector oscillators in one-dimensional and spherical cases are discussed in detail in the next subsections.

The method of solution for the bound states is standard and straightforward. For the resonance states the CCR method is used. The CCR method has been developed to study the autoionizing states also referred to as resonances [30]. Its basic theorem states that the bound state energies of a Hamiltonian do not change under the complex rotation of coordinates,

$$
\boldsymbol{r} \rightarrow \boldsymbol{r} \mathrm{e}^{\mathrm{i} \theta},
$$

whereas the continua move to the complex plane. The discrete eigenvalues appear as the isolated point in the real axis while the points corresponding to the resonances are located in the imaginary plane, either above or below the real axis. After the CCR transformation the Hamiltonian matrix is non-Hermitian and depends on $\theta$. Its eigenvalues are complex and $\theta$ dependent. The $\theta$-dependence of the roots is represented by curves in the complex plane, known as $\theta$-trajectories. For the optimum values of $\theta$ the trajectories are nearly $\theta$-independent. The real part of the corresponding eigenvalue of the CCR Hamiltonian matrix gives the best approximation to the energy of the discrete state and the imaginary part - its width [30]. For the SKG the standard method of diagonalization of the reduced algebraic eigenvalue problem (31) may be used. In the case of the vector KG HO the Schur decomposition method has been employed in this work. Since the matrix $\boldsymbol{M}$ is eigenvaluedependent, the diagonalization is performed by applying at the same time an iterative, self-consistent procedure.

\subsection{One-dimensional oscillators}

Complex coordinate rotated operator (12) reads

$$
\mathrm{W}_{\mathrm{V}}^{\zeta}(\theta)=-\mathrm{e}^{-2 \mathrm{i} \theta} \frac{\mathrm{d}^{2}}{\mathrm{~d} \zeta^{2}}+w_{1} \mathrm{e}^{2 \mathrm{i} \theta} \zeta^{2}-\frac{\lambda}{4} \mathrm{e}^{4 \mathrm{i} \theta} \zeta^{4} .
$$

The Hermite polynomials are a convenient choice for the basis set used in the algebraization procedure. Thus,

$$
\phi_{n}(\zeta)=N_{n} \mathrm{e}^{-\zeta^{2} / 2} H_{n}(\zeta),
$$

where $H_{n}$ are Hermite polynomials and $N_{n}$ is the normalization constant [34]. The set $\left\{\phi_{n}(\zeta)\right\}$ is orthonormal and satisfies the second-order eigenvalue equation

$$
\left(-\frac{\mathrm{d}^{2}}{\mathrm{~d} \zeta^{2}}+\zeta^{2}\right) \phi_{n}(\zeta)=(2 n+1) \phi_{n}(\zeta) .
$$

After some algebra, taking into account the well-known identities for $\zeta^{2} H_{n}(\zeta)$ and $\zeta^{4} H_{n}(\zeta)$ [34] we obtain matrix representation of the operator $(33)$ :

$$
\begin{aligned}
& M_{n^{\prime} n}=\int_{-\infty}^{\infty} \phi_{n}(\zeta) \mathrm{W}_{\mathrm{V}}^{\zeta}(\theta) \phi_{n}(\zeta) \mathrm{d} \zeta= \\
& \sum_{k=-2}^{2} A_{n, k} \delta_{n^{\prime}, n+2 k},
\end{aligned}
$$

where

$$
\begin{aligned}
& A_{n,-2}=-\frac{1}{8} \lambda \mathrm{e}^{4 \mathrm{i} \theta} n(n-1)(n-2)(n-3) \frac{N_{n}}{N_{n-4}}, \\
& A_{n,-1}= \\
& n(n-1)\left(\mathrm{e}^{2 \mathrm{i} \theta} w_{1}-\mathrm{e}^{-2 \mathrm{i} \theta}-\frac{1}{4} \lambda \mathrm{e}^{4 \mathrm{i} \theta}(2 n-1)\right) \frac{N_{n}}{2 N_{n-2}}, \\
& A_{n, 0}= \\
& \frac{1}{2}\left(n+\frac{1}{2}\right)\left(\mathrm{e}^{2 \mathrm{i} \theta} w_{1}+\mathrm{e}^{-2 \mathrm{i} \theta}\right)-\frac{3}{32} \lambda \mathrm{e}^{4 \mathrm{i} \theta}\left(2 n^{2}+2 n+1\right), \\
& A_{n, 1}=\left(\mathrm{e}^{2 \mathrm{i} \theta} w_{1}-\mathrm{e}^{-2 \mathrm{i} \theta}-\frac{1}{4} \lambda \mathrm{e}^{4 \mathrm{i} \theta}(2 n+3)\right) \frac{N_{n}}{8 N_{n+2}}, \\
& A_{n, 2}=-\frac{\lambda}{128} \mathrm{e}^{4 \mathrm{i} \theta} \frac{N_{n}}{N_{n+4}} .
\end{aligned}
$$

To obtain the matrix $\boldsymbol{M}$ in a case of the scalar coupling it is enough to change the sign of $\lambda$ and to set $w_{1} \equiv 1$, $\theta \equiv 0$ in the matrix elements (37). In the non-relativistic limit $(\lambda=0)$ the matrix $\boldsymbol{M}$ becomes the diagonal one for $\theta=0$, whose eigenvalues $w=2 n+1$ correspond to the $2 \times$ energies of the one-dimensional HO.

\subsection{Isotropic oscillators}

Let us consider the VKG described by Eq. (14). By looking for solutions of this equation in the form of power series, one can observe that the power expansion of the radial function $F$ contains only the even powers of $\rho$ [14]. Therefore, we define the variable

$$
\xi=\rho^{2}
$$

and transform operator (15) to the form 


$$
\mathrm{W}_{\mathrm{V}}^{\xi}=-4 \xi \frac{\mathrm{d}^{2}}{\mathrm{~d} \xi^{2}}-2 \frac{\mathrm{d}}{\mathrm{d} \xi}+\frac{l(l+1)}{\xi}+w_{1} \xi-\frac{1}{4} \lambda \xi^{2} .
$$

Introducing the complex rotation

$$
\xi \rightarrow \xi \mathrm{e}^{\mathrm{i} \theta}
$$

we obtain

$$
\begin{aligned}
& \mathrm{W}_{\mathrm{V}}^{\xi}(\theta)=\mathrm{e}^{-\mathrm{i} \theta}\left[-4 \xi \frac{\mathrm{d}^{2}}{\mathrm{~d} \xi^{2}}-2 \frac{\mathrm{d}}{\mathrm{d} \xi}+\frac{l(l+1)}{\xi}\right] \\
& \quad+\mathrm{e}^{\mathrm{i} \theta} w_{1} \xi-\frac{1}{4} \lambda \mathrm{e}^{2 \mathrm{i} \theta} \xi^{2} .
\end{aligned}
$$

Let us define the basis set of Sturmian functions

$$
\phi_{n}(\xi)=N_{n l} \xi^{\frac{l+1}{2}} \mathrm{e}^{-\xi / 2} L_{n}^{(l+1 / 2)}(\xi),
$$

where $l$ is fixed and $L_{n}^{(l+1 / 2)}$ are the generalized Laguerre polynomials of a degree $n=0,1,2, \ldots$ and

$$
N_{n l}=\left(\frac{n !}{\Gamma(n+l+3 / 2)}\right)^{1 / 2} .
$$

Using the well-known properties of the generalized Laguerre polynomials [34], one can find that the Sturmian functions (42) satisfy the second-order differential equation

$$
\begin{gathered}
\left(-4 \xi \frac{\mathrm{d}^{2}}{\mathrm{~d} \xi^{2}}-2 \frac{\mathrm{d}}{\mathrm{d} \xi}+\frac{l(l+1)}{\xi}\right) \phi_{n}(\xi)= \\
(4 n+2 l+3-\xi) \phi_{n}(\xi),
\end{gathered}
$$

the orthogonality condition

$$
\int_{0}^{\infty} \xi^{-1 / 2} \phi_{n^{\prime}}(\xi) \phi_{n}(\xi) \mathrm{d} \xi=\delta_{n^{\prime} n}
$$

and the relation

where

$$
\xi \phi_{n}(\xi)=D_{n l} \phi_{n+1}(\xi)+E_{n l} \phi_{n}(\xi)+D_{n-1, l} \phi_{n-1}(\xi),
$$

$$
D_{n l}=-\sqrt{(n+1)\left(n+l+\frac{3}{2}\right)},
$$

which shall be employed in further calculations. Let us note that the Sturmian functions (42) correspond to the exact solutions of the Schrödinger radial equation for the nonrelativistic spherical HO, energies of which are given by

$$
E_{n l}=2 n+l+\frac{3}{2} .
$$

Finally, matrix elements of the operator (41) defined as

$$
M_{n^{\prime} n}=\int_{-\infty}^{\infty} \xi^{-1 / 2} \phi_{n}(\xi) \mathrm{W}_{\mathrm{V}}^{\xi}(\theta) \phi_{n}(\xi) \mathrm{d} \xi
$$

are given by

$$
\begin{aligned}
& M_{n^{\prime} n}=2 \mathrm{e}^{-\mathrm{i} \theta} E_{n l} \delta_{n^{\prime} n}+\left(\mathrm{e}^{\mathrm{i} \theta} w_{1}-\mathrm{e}^{-\mathrm{i} \theta}\right) K_{n^{\prime} n}^{1 / 2} \\
& -\frac{1}{4} \lambda \mathrm{e}^{2 \mathrm{i} \theta} K_{n^{\prime} n}^{3 / 2},
\end{aligned}
$$

where

$$
K_{n^{\prime} n}^{1 / 2}=D_{n l} \delta_{n^{\prime}, n+1}+E_{n l} \delta_{n^{\prime} n}+D_{n-1, l} \delta_{n^{\prime}, n-1}
$$

and

$$
K_{n^{\prime} n}^{3 / 2}=D_{n^{\prime} l} K_{n^{\prime}+1, n}^{1 / 2}+E_{n^{\prime} l} K_{n^{\prime} n}^{1 / 2}+D_{n^{\prime}-1, l} K_{n^{\prime}-1, n}^{1 / 2} .
$$

Likewise one-dimensional potentials, one obtains the SKG by changing the sign of $\lambda$ and setting $w_{1} \equiv 1, \theta \equiv 0$ in Eq. (50). In the nonrelativistic limit we can simply obtain the energy eigenvalues (48), by taking $\lambda=0$ and $\theta=0$.

\section{Perturbative approach}

In the limit $\lambda \ll 1$, relativistic energies $E$ can be determined perturbatively with respect to the $\lambda$ as the perturbation parameter. Substituting the perturbation expansions

$$
\Psi=\sum_{p=0}^{\infty} \Psi^{(p)} \lambda^{p}, \quad E=\sum_{q=0}^{\infty} E^{(q)} \lambda^{q}
$$

into KG Eqs. (29) and equating appropriate coefficients we obtain a set of the perturbation equations, ordered according to the powers of $\lambda$. For every kind of the potential the zero-order equation corresponds to the Schrödinger equation for the HO. After using the virial theorem, the first- and the second-order energy corrections for the state $\Psi^{(0)} \equiv|n\rangle$, corresponding to the isotropic VKG may be written as

$$
\begin{aligned}
& E_{n \mathrm{~V}}^{(1)}=-\frac{1}{8}\left\langle n\left|r^{4}\right| n\right\rangle \\
& E_{n \mathrm{~V}}^{(2)}=-\frac{1}{8} E_{n}^{(0)} \sum_{k \neq n} \frac{\left\langle k\left|r^{4}\right| n\right\rangle\left\langle n\left|r^{2}\right| k\right\rangle}{E_{n}^{(0)}-E_{k}^{(0)}} \\
& \quad+\frac{1}{4}\left(E_{n}^{(0)}\right)^{2} \sum_{k \neq n} \frac{\left|\left\langle k\left|r^{2}\right| n\right\rangle\right|^{2}}{E_{n}^{(0)}-E_{k}^{(0)}}+\frac{1}{64} \sum_{k \neq n} \frac{\left|\left\langle k\left|r^{4}\right| n\right\rangle\right|^{2}}{E_{n}^{(0)}-E_{k}^{(0)}} \\
& \quad-\frac{1}{2} E_{n}^{(1)} E_{n}^{(0)} .
\end{aligned}
$$

At this point we note that the perturbation method can determine only approximate corrections to the real parts of the resonance energies. For the isotropic SKG the firstand second-order energy corrections read

$$
\begin{aligned}
& E_{n \mathrm{~S}}^{(1)}=-\frac{1}{2}\left(E_{n}^{(0)}\right)^{2}+\frac{1}{8}\left\langle n\left|r^{4}\right| n\right\rangle, \\
& E_{n \mathrm{~S}}^{(2)}=\frac{1}{64} \sum_{k \neq n} \frac{\left|\left\langle k\left|r^{4}\right| n\right\rangle\right|^{2}}{E_{n}^{(0)}-E_{k}^{(0)}}-E_{n}^{(1)} E_{n}^{(0)} .
\end{aligned}
$$

In a similar way we may determine relativistic perturbation energies for the ZHO described by Eq. (28). In the case of 3D oscillator the first-order energy correction has been derived in [8]. By replacing variable $p \rightarrow r$ we may write the first- and the second-order relativistic corrections in the following forms:

$$
\begin{aligned}
& E_{n \mathrm{Z}}^{(1)}=-\frac{1}{8}\left\langle n\left|r^{4}\right| n\right\rangle, \\
& E_{n Z}^{(2)}=\frac{1}{64} \sum_{k \neq n} \frac{\left|\left\langle k\left|r^{4}\right| n\right\rangle\right|^{2}}{E_{n}^{(0)}-E_{k}^{(0)}}+\frac{1}{16}\left\langle n\left|r^{6}\right| n\right\rangle .
\end{aligned}
$$

Let us note that in the case of one-dimensional potentials, the radial variable $r$ should be replaced by the variable $x \in(-\infty, \infty)$. Results of algebraic calculations are summarized in Table I.

\section{Results and discussion}

In this work, we have carried out the detailed analysis of the dynamics of the relativistic spin- 0 particles moving in an external field of the HO-type potentials, using oneand three dimensional electrostatic and scalar parabolic 
potentials. In these cases, the eigenvalue problems have been reduced to pure algebraic tasks, after transforming the KG equations to the matrix forms, using the expansions of wave functions into relevant basis set functions. In the case of VKG, the parameters (energies and widths) of the resonances created by the effective potentials are obtained using the CCR technique. In the framework of the CCR method, the complex energy eigenvalues

$$
E=E_{r}-\mathrm{i} \frac{\Gamma}{2}
$$

correspond to the stationary points of the $\theta$-trajectories in the complex energy plane [30]. The example trajectories are shown in Fig. 2. Introducing the trajectory length, defined as

$$
\gamma(\theta)=\int_{0}^{\theta} \sqrt{\left(\frac{\mathrm{d} E_{r}}{\mathrm{~d} \theta^{\prime}}\right)^{2}+\frac{1}{4}\left(\frac{\mathrm{d} \Gamma}{\mathrm{d} \theta^{\prime}}\right)^{2}} \mathrm{~d} \theta^{\prime},
$$

we can find that the stability of the resonance locations is achieved in the vicinity of stationary points $\theta_{\mathrm{op}}$, being the solutions of the equation

$$
\frac{\mathrm{d} \gamma(\theta)}{\mathrm{d} \theta}=0 \text {. }
$$

The example plot of the function $d \gamma(\theta) / \mathrm{d} \theta$ is displayed in Fig. 3. The accurate value of $\theta_{\text {op }}$ can be determined by solving the nonlinear equation (62) as well as by plotting the logarithmic derivative of the function $\gamma(\theta)$, which discloses the sharp cusp near the $\theta_{\mathrm{op}}$.

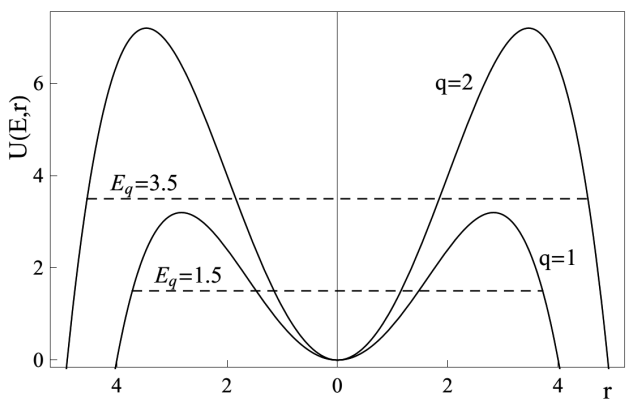

Fig. 1. Effective energy-dependent potentials $q=1,2$ corresponding to levels $E_{1}=1.5$ and $E_{2}=3.5$, respectively. The effective coupling constant $\lambda=0.4$.

As a test, we have employed our numerical procedures to the one-dimensional anharmonic oscillator (AO) given by the Hamiltonian

$$
H_{\mathrm{AO}}=\frac{1}{2} p^{2}+\frac{1}{2} x^{2}-\beta x^{4} .
$$

In Table II the complex resonance energies of the onedimensional AO are displayed and compared with the results obtained on the basis of the operator method [35]. The maximal absolute error of each value does not exceed \pm 1 on the last digits, in all of the tables.

In Table III the lowest four levels of the onedimensional ZHO are listed as functions of the coupling constant $\lambda$.

In Table III the first six levels of the onedimensional SKG and VKG are listed, for several values

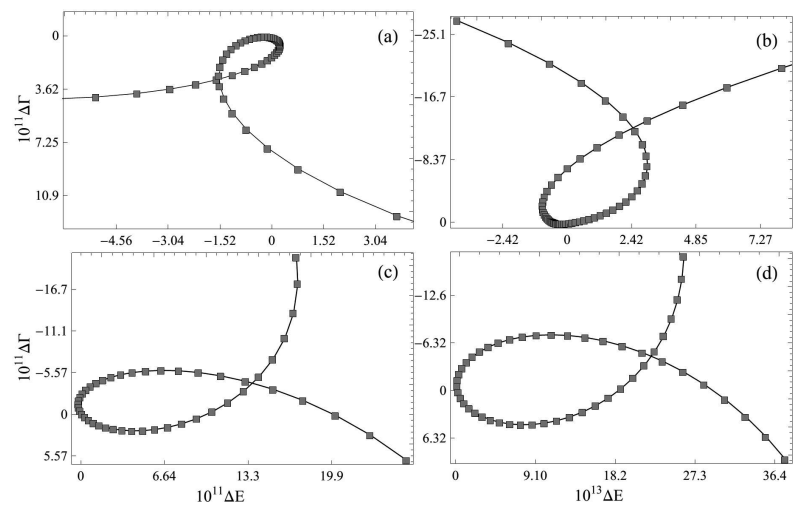

Fig. 2. Complex energy $\theta$-trajectories $\left(\Delta E=E_{r} / E_{0}-\right.$ 1, $\left.\Delta \Gamma=\Gamma / \Gamma_{0}-1\right)$ of the one-dimensional AO, for states: (a) $n=4$, (b) $n=5$ and $\beta=0.1$. Complex energy $\theta$-trajectories for states: (c) $n=4$, (d) $n=5$ and the coupling constant $\beta=0.5$. The values of $E_{0}$ and $\Gamma_{0}$ correspond to the stationary points of trajectories.

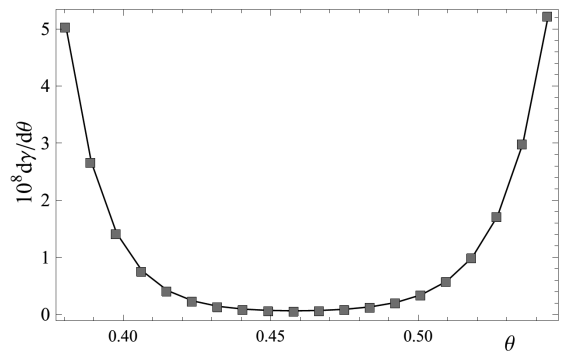

Fig. 3. Dependence of the derivative of the function $\gamma(\theta)$ corresponding to the state $n=4$ of the onedimensional AO with $\beta=0.1$. The zero corresponds to $\theta_{\mathrm{op}}=0.452$.

of the coupling constant $\lambda$. We can see that energies of the ZHO are close to the real parts of the VKG energies. For $\lambda=0.1$ the results are consistent on a level of 2 or 3 decimal figures. For lower $\lambda$, higher accordance is expected. This follows from Table I where perturbation results are presented. The perturbation theory gives equal first-order corrections for ZHO and VKG while the second-order corrections are only slightly different. The same is also observed for isotropic oscillators.

In Table $\mathrm{V}$ the resonance energies and the widths of the 3D isotropic VKG are displayed as functions of the oscillator frequency. One can see that for $\omega<1000$ the imaginary parts of the eigenvalues are negligible in comparison with the real parts. In this region, the resonance energies may be approximated by real bound states energies. Using this approximation we neglect the tunneling by termination of the radial integrals at the radius $r=R_{b}$, which corresponds to the maximum of the effective potential barrier. In the last column, energies $E^{b}$ obtained in the framework of the power series expansion method [14] are displayed. In the latter approach an additional spherical confinement of the radius $R_{b}$ has been introduced to perform the stabilization of the autoionizing resonances. As a consequence, the imaginary parts 
of the complex resonance energies disappear. In this $\omega$ region both methods are in perfect agreement.

Table VI shows the energies of the 3D isotropic SKG and VKG for the states $n \leq 2$ and $l \leq 4$, as functions of $\lambda$.

The application area for the models presented in this paper may be nuclear physics and the QCD [36].
The power-law potentials of the form $V(r)=A+b r^{\nu}$ are broadly accepted in the mass spectra investigations of various quarkonia systems [37]. Some other potentials with vector and scalar couplings have been studied in the context of the diatomic potentials [38].

TABLE I

Relativistic first-and second-order energy corrections $E^{(1)}$ and $E^{(2)}$ corresponding to SKG, VKG and ZHO. Following definitions: $\varepsilon_{n l \mathrm{~S}}^{(2)}=\frac{1}{32}\left(31 n^{3}+3 l^{3}\right)+\frac{n l}{64}(93 n+43 l+136)+\frac{1}{128}\left(279 n^{2}+61 l^{2}\right)$ and $\varepsilon_{n l \mathrm{~V}}^{(2)}=\frac{1}{32}\left(23 n^{3}+l^{3}\right)+\frac{3 n l}{64}(23 n+9 l+$ $32)+\frac{1}{128}\left(207 n^{2}+33 l^{2}\right)$ are used below

\begin{tabular}{c|c|c|c}
\hline \hline & SKG & VKG & ZHO \\
\hline$E_{n}^{(1)}$ & $-\frac{1}{32}\left(10 n^{2}+10 n+1\right)$ & one-dimensional potentials & $-\frac{3}{32}\left(2 n^{2}+2 n+1\right)$ \\
$E_{n}^{(2)}$ & $\frac{1}{512}\left(126 n^{3}+189 n^{2}+37 n-13\right)$ & $-\frac{3}{32}\left(2 n^{2}+2 n+1\right)$ & $\frac{1}{512}\left(46 n^{3}+69 n^{2}+101 n+39\right)$ \\
& & $\frac{1}{512}\left(46 n^{3}+69 n^{2}+37 n+7\right)$ & \\
$E_{n l}^{(1)}$ & $-\frac{1}{16}\left[10(2 n+2 l+3)+\frac{1}{2}(2 l+3)(6 l+7)\right]$ & $-\frac{1}{8}\left[3 n(2 n+2 l+3)+\frac{1}{4}(2 l+3)(2 l+5)\right]$ & $-\frac{1}{8}\left[3 n(2 n+2 l+3)+\frac{1}{4}(2 l+3)(2 l+5)\right]$ \\
$E_{n l}^{(2)}$ & $\varepsilon_{n l S}^{(2)}+\frac{1}{128}\left(\frac{409}{2} n+97 l+\frac{195}{4}\right)$ & $\varepsilon_{n l V}^{(2)}+\frac{1}{128}\left(\frac{313}{2} n+67 l+\frac{159}{4}\right)$ & $\varepsilon_{n l V}^{(2)}+\frac{1}{128}\left(\frac{377}{2} n+83 l+\frac{255}{4}\right)$
\end{tabular}

TABLE II

Resonances parameters $E_{r}$ and $\Gamma$ in Ha as functions of the coupling constant $\beta$ of one-dimensional AO and the comparison with the operator method results $E_{r}^{a}$ and $\Gamma^{a}[35]$. The numbers in brackets are the powers of 10 by which the entries are to be multiplied.

\begin{tabular}{|c|c|c|c|c|c|c|c|}
\hline$\beta$ & State & $E_{r}$ & $\Gamma / 2$ & $N$ & $\theta_{\mathrm{op}}$ & $E_{r}^{a}$ & $\Gamma^{a} / 2$ \\
\hline \multirow[t]{6}{*}{0.025} & 0 & 0.479116818226 & $7.2823867[-6]$ & 34 & 0.372 & 0.479117 & 0.000007 \\
\hline & 1 & 1.38562392863 & $7.7481553[-4]$ & 36 & 0.380 & 1.385667 & 0.000771 \\
\hline & 2 & 2.15723298518 & $2.1984206[-2]$ & 38 & 0.390 & 2.157234 & 0.021984 \\
\hline & 3 & 2.77229261221 & 0.16049881 & 40 & 0.392 & & \\
\hline & 4 & 3.33183365658 & 0.49230095 & 40 & 0.396 & & \\
\hline & 5 & 3.93328149241 & 0.96255479 & 42 & 0.400 & & \\
\hline \multirow[t]{6}{*}{0.1} & 0 & 0.397440629821 & $4.4706148[-2]$ & 36 & 0.443 & 0.397441 & 0.044706 \\
\hline & 1 & 1.09645186727 & $3.3866056[-1]$ & 34 & 0.443 & & \\
\hline & 2 & 1.75258978764 & $9.6935273[-1]$ & 36 & 0.447 & & \\
\hline & 3 & 2.49263538856 & 1.8011218 & 38 & 0.452 & & \\
\hline & 4 & 3.28721142455 & 2.7594569 & 40 & 0.452 & & \\
\hline & 5 & 4.12627756498 & 3.8241057 & 40 & 0.453 & & \\
\hline \multirow[t]{6}{*}{0.5} & 0 & 0.373873916781 & 0.30499025 & 42 & 0.490 & 0.373874 & 0.304990 \\
\hline & 1 & 1.20528560358 & 1.2575002 & 48 & 0.492 & 1.205286 & 1.257500 \\
\hline & 2 & 2.19878584648 & 2.6897011 & 56 & 0.494 & 2.198786 & 2.68970 \\
\hline & 3 & 3.32454304343 & 4.3682036 & 60 & 0.495 & & \\
\hline & 4 & 4.54958499992 & 6.2445312 & 66 & 0.497 & & \\
\hline & 5 & 5.85950089192 & 8.2853397 & 72 & 0.498 & & \\
\hline
\end{tabular}

Energies of the one-dimensional ZHO as functions of $\lambda$.

TABLE III

\begin{tabular}{l|c|c|c|c|c}
\hline \hline$\lambda$ & $n=0$ & $n=1$ & $n=2$ & $n=3$ & $n=4$ \\
\hline 0.001 & 0.499905378 & 1.49952461 & 2.49878283 & 3.49766135 & 4.49616551 \\
0.01 & 0.499070883 & 1.49536013 & 2.48798251 & 3.47698092 & 4.46241259 \\
0.1 & 0.491288900 & 1.45736637 & 2.39240766 & 3.29998374 & 4.18299556 \\
1 & 0.441051722 & 1.23705506 & 1.90992951 & 2.51760364 & 3.07828307
\end{tabular}


TABLE IV

Energies $E_{\mathrm{S}}$ for SKG and resonance parameters $E_{r}$ and $\Gamma$ for VKG in Ha as functions of effective coupling constant $\lambda$ of one-dimensional oscillators. The values of $N$ and $\theta_{\text {op }}$ indicate the size of the basis and the optimal rotation angle corresponding to this accuracy, respectively.

\begin{tabular}{|c|c|c|c|c|c|c|c|}
\hline$\lambda$ & State & $E_{\mathrm{S}}$ & $N$ & $E_{r}$ & $\Gamma / 2$ & $N$ & $\theta_{\mathrm{op}}$ \\
\hline \multirow[t]{6}{*}{0.1} & 0 & 0.4966666614032 & 14 & 0.490740861391 & $2.5349767[-12]$ & 34 & 0.305 \\
\hline & 1 & 1.44032401463 & 14 & 1.45594824022 & $1.6434561[-11]$ & 36 & 0.292 \\
\hline & 2 & 2.33839462096 & 14 & 2.39039162655 & $6.0324606[-11]$ & 38 & 0.280 \\
\hline & 3 & 3.19826567100 & 16 & 3.29753613858 & $1.6512866[-10]$ & 40 & 0.265 \\
\hline & 4 & 4.02548285848 & 18 & 4.18022447116 & $3.7540738[-10]$ & 40 & 0.260 \\
\hline & 5 & 4.82433874665 & 20 & 5.04082434820 & $7.4966981[-10]$ & 44 & 0.250 \\
\hline \multirow[t]{6}{*}{0.2} & 0 & 0.493054567932 & 16 & 0.481610529890 & $1.1074486[-6]$ & 34 & 0.360 \\
\hline & 1 & 1.39031508087 & 18 & 1.41654984202 & $3.9337842[-6]$ & 36 & 0.335 \\
\hline & 2 & 2.21708439057 & 20 & 2.29957185066 & $8.6996191[-6]$ & 36 & 0.312 \\
\hline & 3 & 2.99070437157 & 22 & 3.14031570354 & $1.5409478[-5]$ & 36 & 0.285 \\
\hline & 4 & 3.72229045403 & 26 & 3.94569369156 & $2.3960833[-5]$ & 36 & 0.265 \\
\hline & 5 & 4.41948106379 & 28 & 4.72087075103 & $3.4203993[-5]$ & 38 & 0.251 \\
\hline \multirow[t]{6}{*}{0.4} & 0 & 0.485500590162 & 20 & 0.462791292198 & $6.1410336[-4]$ & 28 & 0.390 \\
\hline & 1 & 1.31014173396 & 22 & 1.34690806717 & $1.2783944[-3]$ & 28 & 0.340 \\
\hline & 2 & 2.04091380364 & 24 & 2.15290561338 & $1.8983627[-3]$ & 30 & 0.320 \\
\hline & 3 & 2.70880932312 & 26 & 2.90229326358 & $2.4580749[-3]$ & 36 & 0.290 \\
\hline & 4 & 3.33065082616 & 30 & 3.60801434263 & $2.9597028[-3]$ & 36 & 0.260 \\
\hline & 5 & 3.91676051403 & 32 & 4.27860775850 & $3.4095914[-3]$ & 44 & 0.260 \\
\hline \multirow[t]{6}{*}{0.6} & 0 & 0.477927963416 & 22 & 0.443890625815 & $4.5734856[-3]$ & 34 & 0.405 \\
\hline & 1 & 1.24760552561 & 24 & 1.28813612873 & $7.2269610[-3]$ & 36 & 0.360 \\
\hline & 2 & 1.91474236567 & 26 & 2.03931624306 & $8.9593064[-3]$ & 36 & 0.320 \\
\hline & 3 & 2.51733167372 & 30 & 2.72793138199 & $1.0179502[-2]$ & 38 & 0.295 \\
\hline & 4 & 3.07438987214 & 32 & 3.37045820745 & $1.1083444[-2]$ & 38 & 0.270 \\
\hline & 5 & 3.59696704476 & 34 & 3.97704925278 & $1.1777406[-2]$ & 46 & 0.242 \\
\hline
\end{tabular}

TABLE V

Resonances energies $E_{r}$ and widths $\Gamma$ in Ha for ground state of the isotropic VKG as functions of the oscillator frequency $\omega$ and comparison with the power series energies $E^{b}$ [14].

\begin{tabular}{c|c|c|c|c|c}
\hline \hline$\omega$ & $E_{r}$ & $\Gamma / 2$ & $N$ & $\theta_{\text {op }}$ & $E^{b}$ \\
\hline 0.1 & 1.49999750385 & & 1 & & 1.49999750385 \\
1 & 1.49997503931 & & 2 & & 1.49997503931 \\
4 & 1.49990016780 & & 2 & & \\
5 & 1.49987521415 & & 2 & & 1.49987521415 \\
10 & 1.49975047229 & & 2 & & 1.49975047229 \\
20 & 1.49950112043 & & 2 & & \\
50 & 1.49875411717 & & 2 & & 1.49875411717 \\
100 & 1.49751260292 & & 2 & & 1.49751260292 \\
200 & 1.49504254359 & & 4 & & \\
400 & 1.49015336360 & $2.48[-53]$ & 38 & 0.448 & \\
500 & 1.48773371791 & $1.351406[-42]$ & 36 & 0.482 & 1.48773371791 \\
1000 & 1.47587484316 & $2.7494179[-21]$ & 30 & 0.584 & 1.47587484316 \\
2000 & 1.45326001561 & $7.6655710[-11]$ & 32 & 0.700 & \\
4000 & 1.41169543662 & $8.1705863[-6]$ & 32 & 0.742 & \\
10000 & 1.30663269335 & $4.7464052[-3]$ & 32 & 0.652 & \\
20000 & 1.19207935056 & $2.5847925[-2]$ & 34 & 0.800 & \\
40000 & 1.06858500487 & $4.4571410[-2]$ & 34 & 0.780 &
\end{tabular}


TABLE VI

Energies $E_{\mathrm{S}}$ for isotropic SKG and resonance parameters $E_{r}$ and $\Gamma$ for isotropic VKG in Ha as functions of effective coupling constant $\lambda$, for several states $(n, l)$.

\begin{tabular}{|c|c|c|c|c|c|c|c|c|}
\hline$\lambda$ & $n$ & $l$ & $E_{S}$ & $N$ & $E_{r}$ & $\Gamma / 2$ & $\theta_{\mathrm{op}}$ & $N$ \\
\hline \multirow[t]{15}{*}{0.1} & \multirow[t]{5}{*}{0} & 0 & 1.44032401463 & 6 & 1.45594824022 & $1.64346[-11]$ & 0.564 & 16 \\
\hline & & 1 & 2.32942137333 & 6 & 2.40053970192 & $3.91777[-11]$ & 0.562 & 16 \\
\hline & & 2 & 3.17412762578 & 8 & 3.32573699904 & $6.14193[-11]$ & 0.538 & 18 \\
\hline & & 3 & 3.98176659289 & 8 & 4.23267527544 & $7.49480[-11]$ & 0.520 & 18 \\
\hline & & 4 & 4.75782094581 & 8 & 5.12240700713 & $7.71105[-11]$ & 0.480 & 18 \\
\hline & \multirow[t]{5}{*}{1} & 0 & 3.19826567099 & 10 & 3.29753613858 & $1.65129[-10]$ & 0.515 & 18 \\
\hline & & 1 & 4.01819853773 & 10 & 4.18903355731 & $2.93594[-10]$ & 0.490 & 18 \\
\hline & & 2 & 4.80438860715 & 10 & 5.06554181977 & $4.01972[-10]$ & 0.465 & 18 \\
\hline & & 3 & 5.56156220108 & 12 & 5.92770154557 & $4.59713[-10]$ & 0.446 & 18 \\
\hline & & 4 & 6.29342185732 & 12 & 6.77613236164 & $4.60331[-10]$ & 0.420 & 18 \\
\hline & \multirow[t]{5}{*}{2} & 0 & 4.82433874662 & 12 & 5.04082434819 & $7.49670[-10]$ & 0.460 & 18 \\
\hline & & 1 & 5.59212711390 & 12 & 5.88911345937 & $1.14254[-9]$ & 0.440 & 20 \\
\hline & & 2 & 6.33298830318 & 12 & 6.72545005181 & $1.44301[-9]$ & 0.420 & 20 \\
\hline & & 3 & 7.05020863046 & 12 & 7.55019992230 & $1.58452[-9]$ & 0.410 & 20 \\
\hline & & 4 & 7.74644764453 & 12 & 8.36373216418 & $1.56048[-9]$ & 0.380 & 20 \\
\hline \multirow[t]{15}{*}{1} & \multirow[t]{5}{*}{0} & 0 & 1.15394569334 & 14 & 1.20292495284 & $2.35728[-2]$ & 0.780 & 30 \\
\hline & & 1 & 1.71507475808 & 16 & 1.91677582400 & $1.53251[-2]$ & 0.712 & 30 \\
\hline & & 2 & 2.20926873750 & 16 & 2.57112789428 & $8.50659[-3]$ & 0.652 & 30 \\
\hline & & 3 & 2.66017387395 & 18 & 3.17868590387 & $4.37938[-3]$ & 0.590 & 30 \\
\hline & & 4 & 3.07986733340 & 18 & 3.74888519570 & $2.15794[-3]$ & 0.540 & 30 \\
\hline & \multirow[t]{5}{*}{1} & 0 & 2.26092439489 & 18 & 2.48882076584 & $2.49759[-2]$ & 0.645 & 30 \\
\hline & & 1 & 2.72705785648 & 18 & 3.07456468745 & $1.93057[-2]$ & 0.600 & 30 \\
\hline & & 2 & 3.15582182392 & 20 & 3.63535078156 & $1.29483[-2]$ & 0.560 & 30 \\
\hline & & 3 & 3.55719049268 & 20 & 4.17232484554 & $7.92841[-3]$ & 0.510 & 30 \\
\hline & & 4 & 3.93724437013 & 22 & 4.68738439127 & $4.55485[-3]$ & 0.462 & 32 \\
\hline & \multirow[t]{5}{*}{2} & 0 & 3.18843658067 & 20 & 3.57922995532 & $2.49540[-2]$ & 0.560 & 32 \\
\hline & & 1 & 3.60263524814 & 22 & 4.09546264691 & $2.07679[-2]$ & 0.520 & 32 \\
\hline & & 2 & 3.99163772291 & 24 & 4.59830080428 & $1.53731[-2]$ & 0.460 & 32 \\
\hline & & 3 & 4.36085916330 & 24 & 5.08708335380 & $1.04453[-2]$ & 0.400 & 32 \\
\hline & & 4 & 4.71396623992 & 26 & 5.56182567162 & $6.64300[-3]$ & 0.368 & 32 \\
\hline
\end{tabular}

\section{Acknowledgments}

The author thanks Professor J. Karwowski for valuable discussions and positive suggestions.

\section{References}

[1] A. Beléndez, D.I. Méndez, M.L. Alvarez, C. Pascual, T. Beléndez, Int. J. Mod. Phys. B 23, 521 (2009).

[2] K. Nikolsky, Z. Phys. 62, 677 (1930).

[3] I. Postępska, Acta Phys. Pol. 4, 269 (1935).

[4] P.A. Cook, Lett. Nuovo Cimento 10, 419 (1971).

[5] A.L. Harvey, Phys. Rev. D 6, 1474 (1972).

[6] M. Sachs, Lett. Nuovo Cimento 13, 169 (1975).

[7] M. Moshinsky, A. Szczepaniak, J. Phys. A Math. Gen. 22, L817 (1989).

[8] M. Znojil, J. Phys. A Math. Gen. 29, 2905 (1996).

[9] F.M. Toyama, Y. Nogami, Phys. Rev. A 59, 1056 (1999).
[10] LMC R. Lisboa, M. Malherio, A.S. de Castro, P. Alberto, M. Fiolhais, Phys. Rev. C 69, 024319 (2004).

[11] J. Karwowski, G. Pestka, Theoret. Chem. Account 118, 519 (2007).

[12] A. Rao Nagalakshim, B.A. Kagali, Phys. Scr. 77, 015003 (2008).

[13] I. Bars, Phys. Rev. D 79, 045009 (2009).

[14] A. Poszwa, Phys. Rev. A 82, 052110 (2010).

[15] A. Boumali, A. Haldallah, A. Toumi, Phys. Scr. 84, 037001 (2011).

[16] A.S. Larkin, V.S. Filinov, Phys. Lett. A 377, 1171 (2013).

[17] J. Benítez, R.P. Martnez y Romero, H. N. NúezYépez, A.L. Salas-Brito Phys. Rev. Lett. 64, 1643 (1990).

[18] P. Rozmej, R. Arveu, J. Phys. A Math. Gen. 32, 5367 (1999)

[19] M.H. Pacheco, R.R. Landim, C.A.S. Almeida, Phys. Lett. A 311, 93 (2003). 
[20] W. Chunfeng, X. Kang, Int. J. Theor. Phys. 43, 2395 (2004).

[21] R. Rekioua, T. Boudjedaa, Eur. Phys. J. C 49, 1091 (2007).

[22] A. Bermudez, M.A. Martin-Delgado, E. Solano, Phys. Rev. A 76, 041801(R) (2007).

[23] R. De Lima Rodrigues, Phys. Lett. A 372, 2587 (2008).

[24] N.A. Rao, B.A. Kagali, Phys. Scr. 77, 015003 (2008).

[25] A. Boumali, A. Halfdallah, A. Toumi, Phys. Scr. 84 , 037001 (2011).

[26] A.D. Alhaidari, H. Bahlouli, A. Al-Hasan, Phys. Lett. A 349, 87 (2006).

[27] M.K. Bahar, F. Yasuk, Adv. High Energy Phys. 2013, 814985 (2013).

[28] W. Greiner Relativistic Quantum Mechanics, Springer-Verlag, Berlin (2000).
[29] G.E. Brown, D.G. Ravenhall, Proc. R. Soc. London, Ser. A 208, 552 (1952).

[30] N. Moiseyev Non-Hermitian Quantum Mechanics, Cambridge University Press, New York 2011.

[31] R.A. Weder, J. Math. Phys. 15, 20 (1974).

[32] P. Seba, Lett. Math. Phys. 16, 51 (1988).

[33] G. Pestka, M. Bylicki, J. Karwowski, J. Phys. B At. Mol. Opt. Phys. 39, 2979 (2006).

[34] M. Abramowitz, I.A. Stegun Handbook of Mathematical Functions Dover, New York 1968.

[35] I.D. Feranchuk, L.I. Komarov, I.V. Nichipor, A.P. Ulyanenkov, Ann. Phys.-New York 238, 370 (1995).

[36] J.N. Ginnocchio, Phys. Rev. Lett. 78, 436 (1997).

[37] M.M. Panja, R. Dutt, Y.P. Varshini, J. Phys. A Math. Gen. 22, 2991 (1989).

[38] H. Akcay, R. Sever, Phys. Scr. 89, 015003 (2014). 